\title{
Combined Pyogenic and Tuberculous Osteomyelitis of the Clavicle: A Case Report
}

\author{
Jairam D Jagiasi ${ }^{1}$, Mohit R Upadhyaya ${ }^{1}$, Parth K Mehta ${ }^{1}$ \\ Learning Point of the Article: \\ A high degree of suspicion is required to diagnose the underlying cause of unusual presentation of osteomyelitis.
}

\section{Abstract}

Introduction: Tuberculous osteomyelitis of the clavicle accounts for 1-3\% of cases of osteoarticular tuberculosis. It presents with non-specific symptoms and may have superadded infections with pyogenic organisms, requiring a high degree of suspicion to adequately diagnose and initiate appropriate treatment.

Case Report: We describe a case of a 35-year-old male with osteolytic clavicular lesion and abscess in the supraclavicular fossa. Tissue diagnosis revealed tuberculous osteomyelitis with superadded infection with Staphylococcus aureus. He was managed with debridement and antitubercular therapy.

Conclusion: A high degree of suspicion is required to adequately test and diagnose the cause of osteomyelitis of the clavicle, in the absence of a predisposing event, as staphylococcal and tuberculous infection can present simultaneously.

Keywords: Clavicle, tuberculosis, osteomyelitis.

\section{Introduction}

Tuberculous osteomyelitis of the clavicle accounts for 1-3\% of cases of osteoarticular tuberculosis (TB). Most cases involve a primary pulmonary or osteoarticular lesion [1]. It affects children more commonly than adults and involves the medial end if the clavicle and the sternoclavicular joint [2]. It presents with non-specific symptoms such as fever, malaise, and an osteolytic bone lesion, making it easy to misdiagnose as a cystic bone tumor or metabolic bone disease $[3,4]$. We report this case due to its unusual presentation of showing positive culture reports for both pyogenic and tuberculous organisms.

\section{Case Report}

A 35-year-old male was referred with high-grade fever, a $5 \mathrm{~cm} \times$ $5 \mathrm{~cm}$ fluctuant swelling in the supraclavicular fossa extending into anterior chest wall (Fig. 1) and an osteolytic lesion $1 \mathrm{~cm} \times 1$ $\mathrm{cm}$ in size, in the middle third of the right clavicle (Fig. 2), incidentally found on chest radiograph. The abscess had developed slowly over 1 month, but he developed fever over 3 days. The patient had no prior history of TB or any procedure done in this region. Contrast-enhanced magnetic resonance imaging (MRI) of the clavicle was done to determine extent of bony infection. MRI was reported as an osteolytic focus in the middle one-third of the right clavicle with thinning and erosion of the cortex, associated with heterogeneously enhancing periosseous soft tissues and non-enhancing foci/abscesses. These findings suggested an infective etiology (osteomyelitis). Pre-operative hemoglobin was $10.1 \mathrm{~g} / \mathrm{dl}$, leukocyte count was $14,800 / \mathrm{mm} 3$, erythrocyte sedimentation rate was $72 \mathrm{~mm} / \mathrm{h}$, and C-reactive protein was positive. Patient was HIV, hepatitis B and hepatitis $C$ negative. Chest radiograph showed a heterogeneous opacity in the right middle lobe of the lung. Due to picture of acute abscess formation, decision of primary surgical debridement of the focus was taken.

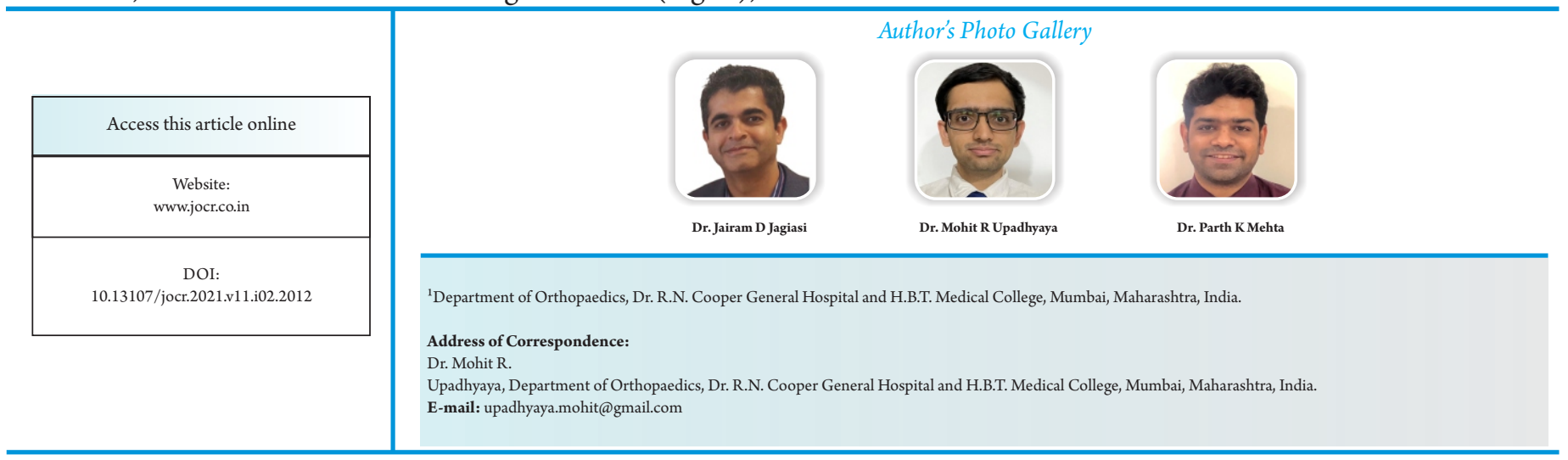

Journal of Orthopaedic Case Reports | pISSN 2250-0685 | eISSN 2321-3817 | Available on www.jocr.co.in | doi:10.13107/jocr.2021.v11.i02.2012 This is an Open Access article distributed under the terms of the Creative Commons Attribution Non-Commercial License (http://creativecommons.org/licenses/by-nc/3.0) which permits unrestricted non-commercial use, distribution, and reproduction in any medium, provided the original work is properly cited. 


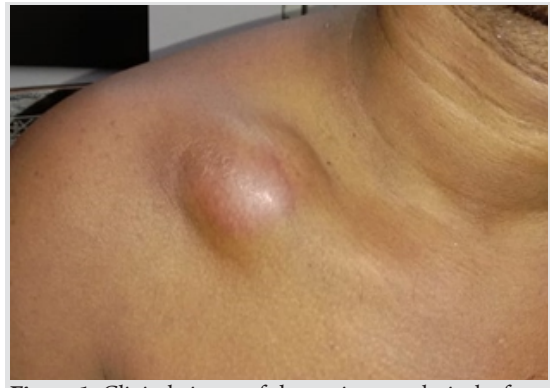

Figure 1: Clinical picture of abscess in supraclavicular foss and anterior chest wall.

The patient underwent drainage of the abscess and debridement of the clavicle under general anesthesia. The clavicle showed a defect of $1 \mathrm{~cm} \times 0.5 \mathrm{~cm}$ in the superior and inferior cortex (Fig. 3-5). Samples of pus and sequestered bone were sent for Gram staining, culture sensitivity, GeneXpert, TB culture, and staining. The patient was started on antibiotics empirically till culture reports were available.

The patient was referred to chest medicine for opacity in chest radiograph and was diagnosed as pulmonary $\mathrm{TB}$ and advised anti-Koch's therapy for the same.

The pus culture revealed growth of methicillin sensitive Staphylococcus aureus, histopathology showed granulomatous inflammation, while GeneXpert and TB culture showed Mycobacterium tuberculosis, sensitive to rifampicin. Blood culture showed no growth. Based on these reports, the patient was started on injectable amoxicillin + clavulanic acid $1.2 \mathrm{~g}$ twice daily for week along with anti-Koch's therapy for 6 months with rifampicin, isoniazid, ethambutol, and pyrazinamide for 2 months and 4 months of rifampicin, isoniazid, and ethambutol. The patient was given a universal shoulder immobilizer to protect the clavicle. Pendulum exercises and elbow range were started at 2 weeks post-operative. Shoulder range of motion exercises were allowed after 4 weeks. Lifting heavy objects were not allowed till osseous healing was seen.

The surgical wound healed uneventfully after 15 days. The osseous lesion showed signs of radiological healing at 2 months follow-up, with complete resolution at 5 months follow-up (Fig. 6). At 9 months follow-up, the patient had no complaints and was able to use his arm comfortably to perform overhead activities, including lifting heavy objects (Fig. 7).

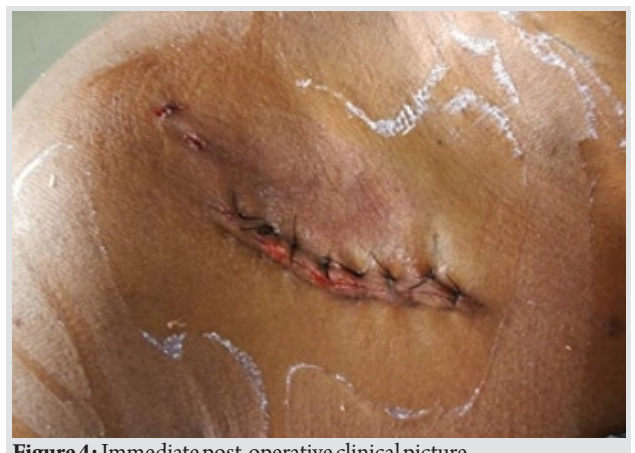

Figure 4: Immediate post-operative clinical picture.

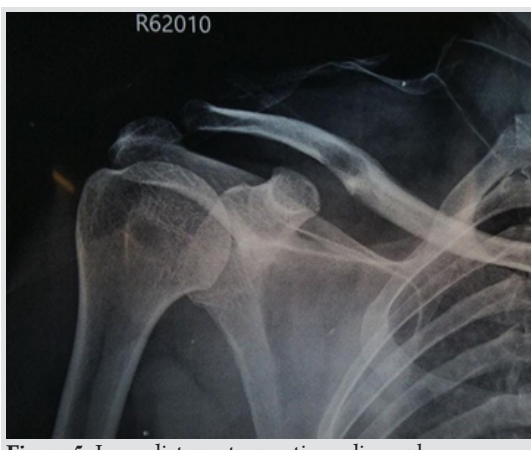

Figure 5: Immediate post-operative radiograph

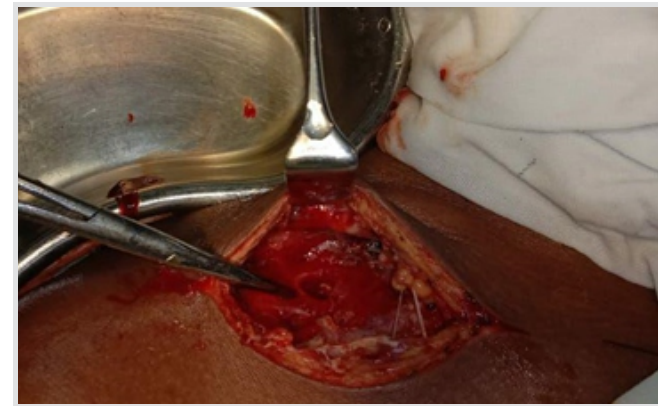

Figure 3: Intraoperative image showing cortical defect in clavicle.

Discussion

Osteomyelitis of the clavicle is an extremely rare entity [5]. In adults, it is seen as a complication secondary to head-and-neck surgery, fracture fixation, and subclavian catheterization $[6,7]$. In the absence of such predisposing factors, the patient should be further investigated for conditions that cause immunosuppression, allowing for hematogenous spread of the infection from another primary focus.

TB is still highly prevalent in India, affecting almost 2.7 million people [8]. It is the most common differential diagnosis for osteomyelitis in India. TB is also known to cause immunosuppression [9], increasing the susceptibility of the host to other infections. Osteoarticular TB is a paucibacillary condition and may be associated with normal chest radiographs and a negative tuberculin skin test [10], making tissue culture necessary for diagnosis and initiating proper treatment.

Staphylococcus coinfection can mask the symptoms of tuberculous osteomyelitis and cause a delay in diagnosis [11]. Simply managing such a case with antibiotics for staphylococcal osteomyelitis may lead to the infection appearing to settle initially, but the underlying TB would go untreated causing repeated abscess, sinuses, and continued destruction of the bone [12]. The infection may progress, affecting the sternoclavicular or acromioclavicular and may necessitate excision of a large section of the sequestered clavicle [13], which may prove to be troublesome who require to lift heavy objects overhead. Early diagnosis and adequate anti-Koch's therapyleads to complete resolution of the infection. 


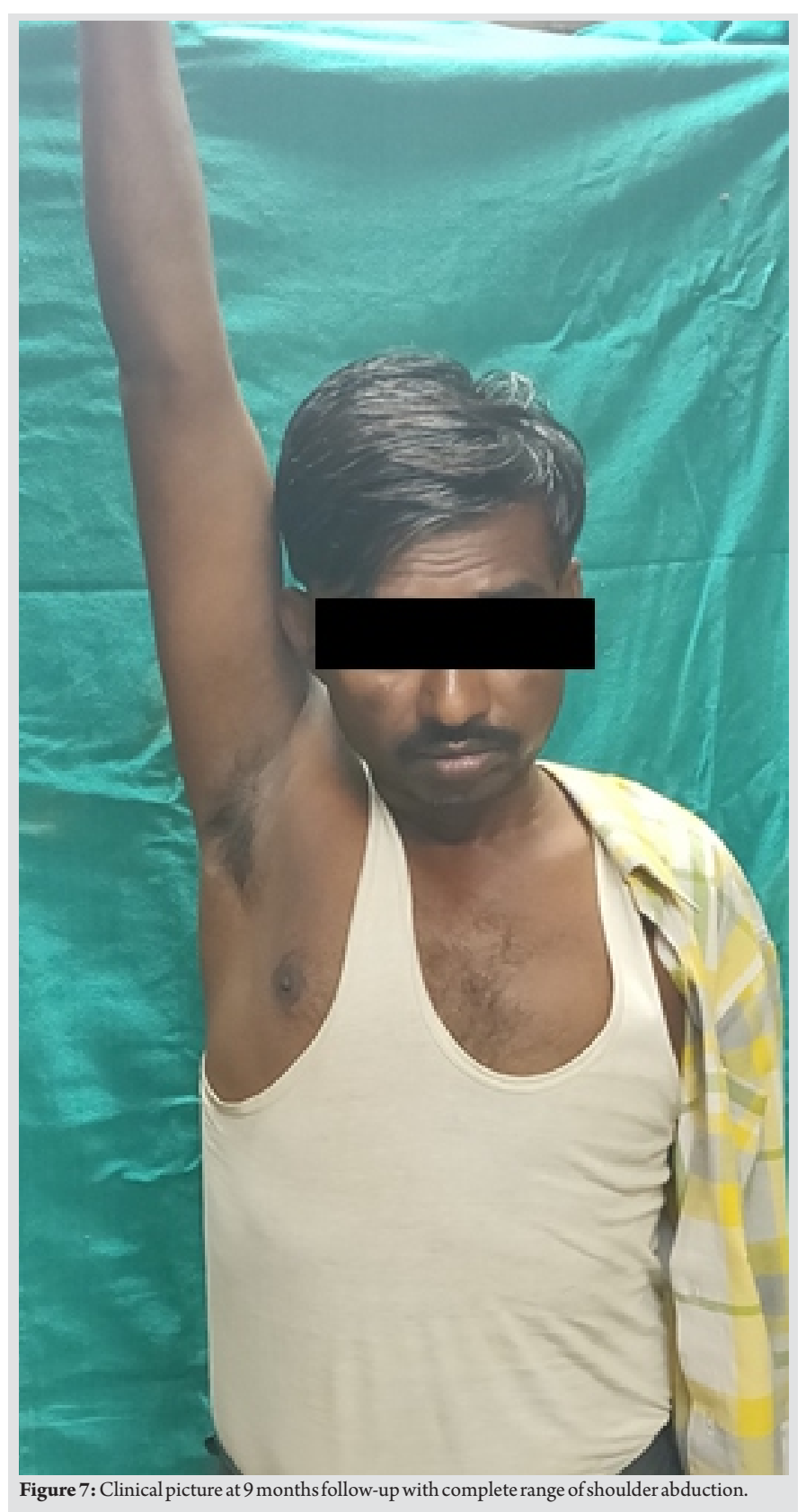

\section{Conclusion}

A high degree of suspicion is required to adequately test and diagnose the cause of osteomyelitis of the clavicle as staphylococcal and tuberculous infection can present simultaneously. In the absence of a predisposing event, a cause for immunosuppression allowing hematogenous spread of infection must be looked for.

\section{Clinical Message}

Coinfection with pyogenic organism and mycobacteria can be found in uncommon sites of osteomyelitis like clavicle. Awareness and vigilance are required to adequately diagnose and treat such cases.

References

1. Verma S, Mehra M. Isolated clavicle bone tuberculosis. Intern J Orthop Surge 2007;8.

2. Clinical Characteristics and Treatment of Clavicle Osteomyelitis: An Over review of 188 Reported Cases. Orthopaedic Proceedings. Available from: https://www.online.boneandjoint.org.uk/doi/abs/10.13 02/1358-992X.2017.22.023. [Last accessed on $2020 \mathrm{Jul}$ 03].

3. Cheng J, Feng S, Lei H, Huo W, Feng H. Tuberculosis of acromioclavicular joint: A case report. BMC Infect Dis 2019;19:111.

4. Prakash J, Aggarwal S, Mehtani A. Primary tuberculosis of the clavicle. Orthopedics 2014;37:e879-84.

5. Clinical Characteristics and Treatment of Clavicle Osteomyelitis: An Over Review of 188 Reported Cases Orthopaedic Proceedings. Available from: https://www.online.boneandjoint.org.uk/doi/abs/10.13 02/1358-992X.2017.22.023. [Last accessed on $2020 \mathrm{Jul}$ $05]$.

6. Alessi DM, Sercarz JA, Calcaterra TC. Osteomyelitis of the clavicle. Arch Otolaryngol Head Neck Surg 1988;114:1000-2.

7. Lee YH, Kerstein MD. Osteomyelitis and septic arthritis. A complication of subclavian venous catheterization. $\mathrm{N}$ Engl 
JMed 1971;285:1179-80.

8. TB Statistics India-latest 2020 Numbers TBFacts. Available from: https://tbfacts.org/tb-statistics-india. [Last accessed on 2020 Jul 05].

9. Roberts T, Beyers N, Aguirre A, Walzl G. Immunosuppression during active tuberculosis is characterized by decreased interferon $-\gamma$ production and CD25 expression with elevated forkhead box p3, transforming growth factor- $\beta$, and interleukin- 4 mRNA levels.J Infect Dis 2007; 195:870-8.

10. Procopie I, Popescu EL, Huplea V, Pleșea RM, Ghelase Ș M, Stoica GA, et al. Osteoraticular tuberculosis-brief review of clinical morphological and therapeutic profiles. Curr Health SciJ 2017;43:171-90.

11. Zayani CB, Daoued L, Tekaya R, Bouebdella M, Abdelmoula L, Kooli M, et al. Tuberculous osteomyelitis masked by staphylococcal infection. Joint Bone Spine 2009;76:429.

12. Epperla N, Kattamanchi S, Fritsche TR. Appearances are deceptive: Staphylococcus super infection of clavicular tuberculous osteomyelitis. Clin Med Res 2015;13:85-8.

13. Agarwal A, Maheshwari R. Tubercular osteomyelitis clavicle: A case report.J Orthop Case Rep 2014;4:51-513.

\section{How to Cite this Article}

Jagiasi JD, Upadhyaya MR, Mehta PK. Combined Pyogenic and Tuberculous Osteomyelitis of the Clavicle: A Case Report. Journal of Orthopaedic Case Reports 2021 February; 11(2):29-32. 